\title{
Chemoradiation-induced superior vena cava syndrome: A case report
}

\author{
Mathieu Castonguay MD ${ }^{1}$, George Rodrigues MD MSc ${ }^{2,3}$, Mark Vincent MD ${ }^{3,4}$, \\ Richard A Malthaner MD MSc ${ }^{3,5}$, Lin-Ruo Guo MD 6
}

M Castonguay, G Rodrigues, M Vincent, RA Malthaner, L-R Guo. Chemoradiation-induced superior vena cava syndrome: A case report. Can Respir J 2008;15(8):444-446.

A case of a 54-year-old man who developed superior vena cava syndrome secondary to vascular fibrosis, 30 months after radical chemoradiation for stage III non-small cell lung cancer, is presented. The literature regarding the etiology, diagnosis and treatment of this rare entity is discussed.

Key Words: Late toxicity; Non-small cell lung cancer; Radiation therapy; Superior vena cava syndrome

Quperior vena cava syndrome (SVCS) is a clinical entity of $\checkmark$ signs and symptoms resulting from impairment of venous blood flow through the superior vena cava (SVC) and into the right atrium of the heart. Pathophysiologically, extrinsic compression of the SVC occurs due to a thin-walled SVC combined with a low intravascular pressure within the SVC itself. The SVC obstruction can ultimately result in increases in venous pressure leading to retrograde flow in collateral veins and interstitial edema in the arm, head and neck regions. Typical signs and symptoms can include facial, neck and upper extremity edema, shortness of breath, cough, hoarseness, neck vein distention and thoracic collateral vein formation. The majority of SVCS cases (more than $80 \%$ ) are caused by malignancies; however, nonmalignant SVCS can occur (1). We present a case of nonmalignant SVCS arising 2.5 years after the completion of radical chemoradiation for stage III non-small cell lung cancer.

\section{CASE PRESENTATION}

A 54-year-old man with a 32 pack-year history of smoking presented with chest pain and hemoptysis that began one month previously. A chest radiograph demonstrated a right upper lobe mass with right upper lobe bronchial obstruction. Computed tomography (CT) of the thorax confirmed a $5 \mathrm{~cm}$ right upper lobe mass involving the right hilum and occlusion of the right main stem bronchus with no mediastinal lymph node enlargement. The primary tumour extended into the mediastinum, and there was external compression of the carina. The SVC was normal during this staging CT. Bronchoscopic biopsy of the right upper lobe lesion demonstrated an adenocarcinoma of the lung, and mediastinoscopy confirmed that the primary tumour invaded into the mediastinum with ipsilateral paratracheal nodal involvement.

\section{Le syndrome de compression de la veine cave supérieure induit par chimioradiothérapie : Un rapport de cas}

Est présenté le cas d'un homme de 54 ans qui a développé un syndrome de compression de la veine cave supérieure secondaire à la fibrose kystique 30 mois après une chimioradiothérapie radicale en traitement d'un cancer du poumon non à petites cellules de stade III. Les publications portant sur l'étiologie, le diagnostic et le traitement de cette entité rare sont exposées.

\begin{abstract}
Metastatic workup with head and bone CT scan was negative; thus, the patient was staged as T4N2M0, stage IIIB.
\end{abstract}

Patient management consisted of neoadjuvant chemotherapy followed by concurrent chemoradiation, in which the patient received a total of four cycles (two cycles induction, two cycles concurrent) of vinblastine and cisplatin, and $60 \mathrm{~Gy}$ of radiation to the primary tumour in 30 fractions using threedimensional conformal radiotherapy. Maximum cord dose was 4500 cGy, V20Gy bilateral lung parameter was 25\%, and the planning target volume treated was $474 \mathrm{~mL}$; all within acceptable institutional levels. The concurrent chemoradiation phase of therapy was interrupted after seven radiation fractions for three weeks due to development of an acute inferior wall myocardial infarction. The patient was diagnosed with doublevessel disease (subtotal proximal right coronary and 70\% left anterior descending occlusions on angiography). The patient was treated with coronary angioplasty and stenting after which the patient completed both the planned chemotherapy and radiation without further protocol modification.

A positron emission tomography scan performed three months after treatment completion demonstrated no uptake. On follow-up at six months, a chest CT demonstrated that the patient had a complete response to therapy. Radiation fibrosis associated with high-dose radiation volume in the right upper lobe was consistently seen on chest $\mathrm{x}$-ray and CT follow-up from six to 30 months (Figures 1 and 2). Eighteen months after treatment completion, the patient presented with hemoptysis. Bronchoscopy demonstrated narrowing of the bronchus intermedius and right upper lobe bronchus, as well as bleeding at the carina consistent with postradiation change. No endobronchial lesion was detected, and biopsies of the abnormal mucosa at the minor carina and anterior trachea were performed. These

\footnotetext{
${ }^{1}$ Department of Pathology, The University of Western Ontario; ${ }^{2}$ Department of Radiation Oncology, London Regional Cancer Program;

${ }^{3}$ Department of Oncology, The University of Western Ontario; ${ }^{4}$ Department of Medical Oncology, London Regional Cancer Program;

${ }^{5}$ Division of Thoracic Surgery and Surgical Oncology, Department of Surgery; ${ }^{6}$ Division of Cardiac Surgery, Department of Surgery, The

University of Western Ontario, London, Ontario

Correspondence: Dr George Rodrigues, London Regional Cancer Program, 790 Commissioners Road East, London, Ontario N6A 4 L6.

Telephone 519-685 8500 ext 52833,fax 519-685-8736, e-mail george.rodrigues@lhsc.on.ca
} 


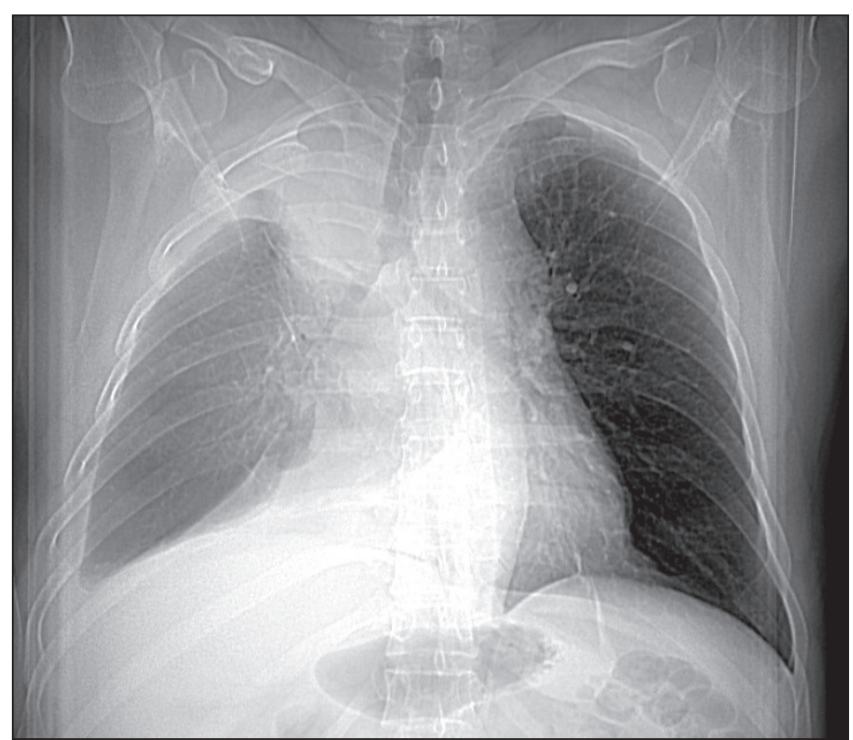

Figure 1) Right upper lung fibrosis on chest $x$-ray

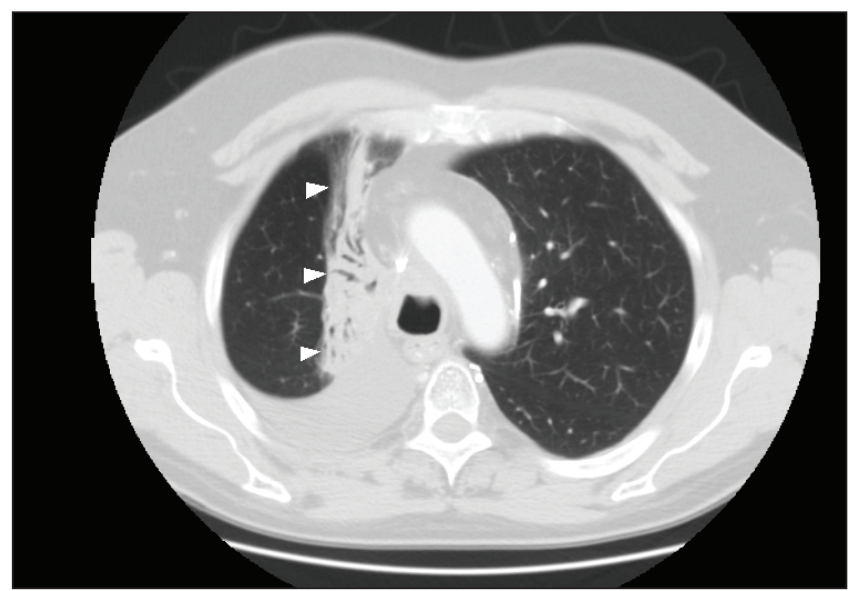

Figure 2) Computed tomography scan of the thorax showing right lung fibrosis. The lateral edge of fibrosis, consistent within the radiation port, is outlined by arrowheads

biopsies did not demonstrate any evidence of recurrent cancer. Therefore, the patient was placed on continued follow-up.

At 30 months of follow-up, the patient presented with progressive facial and neck swelling, nocturnal cough and hoarseness. Clinical examination revealed significant facial swelling and collateral thoracic and abdominal veins. Repeat CT scan showed pinpoint narrowing of the SVC (consistent with radiation fibrosis as in previous high-dose radiation field without evidence of recurrent disease) with numerous collateral veins, large right pleural effusion, right upper lobe lung collapse and fibrosis, and pericardial effusion (Figure 3). Bronchoscopy with biopsy and diagnostic thoracentesis were both negative for tumour recurrence. An echocardiogram demonstrated normal ventricular function, mild aortic sclerosis and moderate pericardial effusion. An SVC cavogram confirmed there was complete occlusion of the SVC with associated collateral veins (Figure 4). No associated thrombosis was observed.

Incidentally, a restaging magnetic resonance imaging of the brain detected an asymptomatic $7 \mathrm{~mm}$ brain metastasis located in the posterior aspect of the frontal lobe, and a second $6 \mathrm{~mm}$

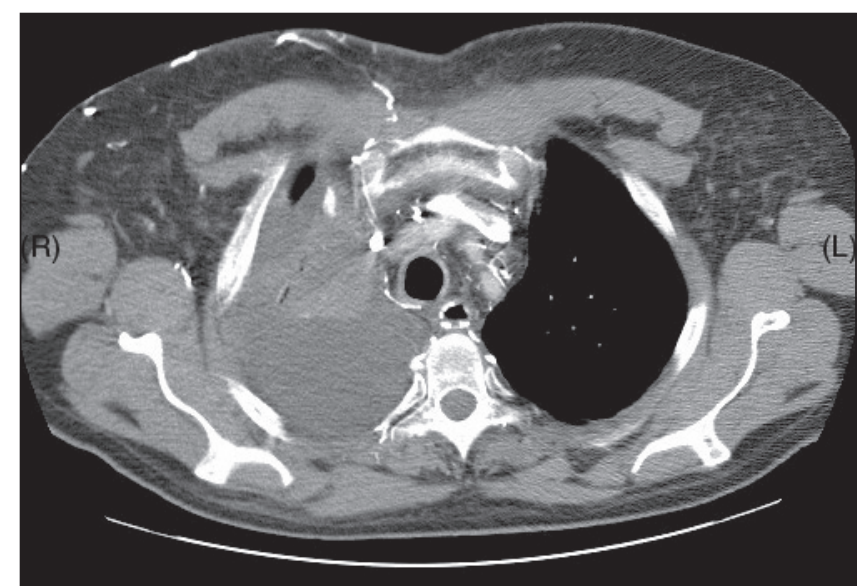

Figure 3) Computed tomography scan of the chest demonstrating right upper lung collapse, large right pleural effusion, collateral veins and narrowing of the superior vena cava. L Left side; $R$ Right side

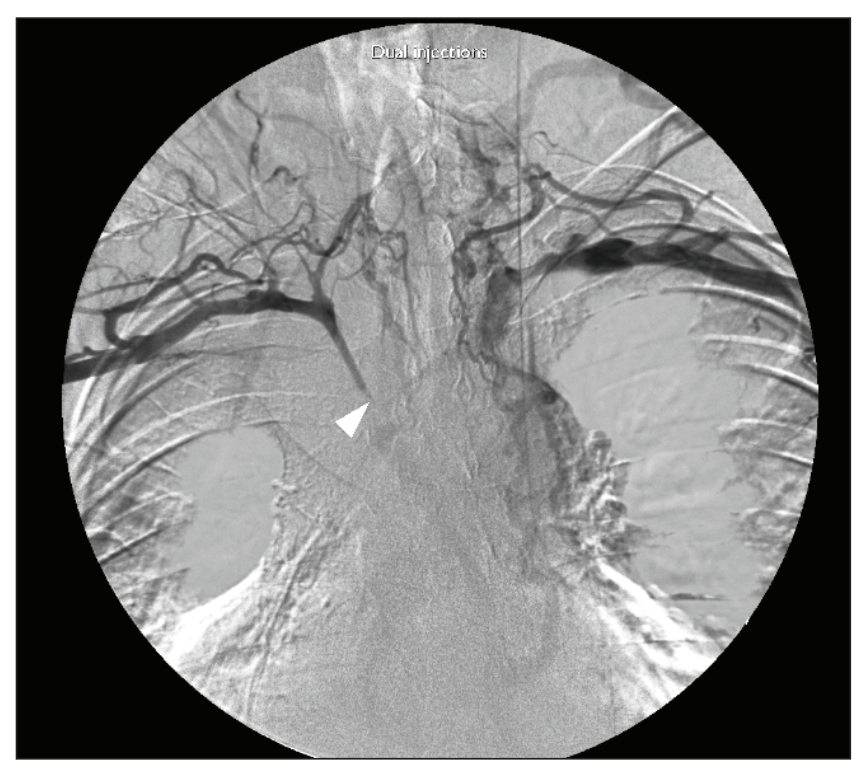

Figure 4) Bilateral venogram demonstrating narrowing of the superior vena cava (arrowhead)

abnormality in the cerebellar vermis consistent with a metastatic deposit. The patient was treated with external beam radiation therapy to the whole brain (30 Gy in 10 fractions with concomitant helical tomotherapy boost to a total of 45 Gy in 10 fractions). For management of the SVCS, the patient underwent autologous spiral saphenous vein bypass grafting between the innominate vein and right atrium. The spiral vein graft was sheathed in a $12 \mathrm{~mm}$ ring-reinforced GoreTex (WL Gore \& Associates, USA) graft to prevent external compression or kinking. Single-vessel coronary artery bypass grafting was also performed because of the preoperative angiogram demonstrating total occlusion of posterior descending coronary artery. A pigmented mediastinal lymph node was excised, and a moderate pericardial effusion was drained intraoperatively; both were negative for malignancy. Extensive mediastinal fibrosis without recurrent tumour was observed during the operative procedure. The patient subjectively improved after surgery; however, three months later, the patient was admitted to a local hospital with dyspnea hypothesized to be due 
to new-onset arrhythmia. A chest x-ray was performed and did not demonstrate any interval change from imaging performed six months previously. He died two weeks later and autopsy examination was not performed.

\section{DISCUSSION}

Cancer-related SVCS makes up the majority of cases in modern SVCS series. Small cell lung cancer, non-small cell lung cancer and Hodgkin's lymphoma are the most frequent cancers associated with this entity. Common nonmalignant causes of SVCS include mediastinal fibrosis (idiopathic versus histoplasmosis), as well as various iatrogenic medical device-related causes leading to thrombosis (eg, pacemaker leads, central venous catheter). Other rare benign causes of SVCS include thyroid goitre, sarcoidosis and tuberculosis. Vascular fibrosis should also be included in the differential diagnosis of nonmalignant SVCS in patients who previously received cancer chemotherapy and radiotherapy and are in clinical remission.

Vascular injury is well established as a consequence of radiation therapy; unfortunately, injuries to the venous system have not been as well studied. Notwithstanding this, veins are believed to be the least radiosensitive blood vessels (2). Radiation-induced fibrosis is attributed to the activation and proliferation of fibroblasts that secrete a disorganized extracellular matrix $(3,4)$. Transforming growth factor-beta is believed to be the key cytokine for both the initiation and perpetuation of radiation-induced fibrosis, leading to recruitment and activation of fibroblasts. Immediately following radiation, transforming growth factor-beta is released by platelets; subsequently, it is released by myofibroblasts, leading to a vicious cycle of fibrosis $(3,4)$. On a molecular level, the generation of reactive oxygen species by radiation is believed to overwhelm local antioxidant defenses, and thereby effect fibrogenic changes (3).

Reports of chemotherapy-induced (5) and radiation therapyinduced (6-8) SVCS (RI-SVCS) are rare in the medical literature. A total of four reported cases of RI-SVCS were identified in the world literature. Van Putten et al (6) report on two cases of RI-SVCS. One case of RI-SVCS occurred in a postpneumonectomy lung cancer patient receiving 60 Gy in 30 fractions of external beam radiation for isolated mediastinal recurrence. RI-SVCS occurred five years after radiation therapy and was treated by SVC stenting. The second patient received radiation therapy for a recurrent maxillary sinus carcinoma to the cervical lymph nodes ( 50 Gy in 25 fractions to the neck and superclavicular regions, with an electron boost of 20 Gy in 10 fractions to sites of gross cervical adenopathy). RI-SVCS with fibrosis occurred seven years after radiation therapy and was successfully treated conservatively with anticoagulation alone. Lee et al (7) report on one case of postoperative radiation therapy for an anterior mediastinal germ cell tumour to a dose of 32 Gy delivered by cobalt-60. RI-SVCS occurred 20 years after radiation therapy and was managed by forming an anastomosis between the left internal jugular vein and the right atrium with the use of a $10 \mathrm{~mm}$ ringed polytetrafluoroethylene graft. Dhaliwal et al (8) report one case of a man who received radiation (unspecified dose) for an unresectable malignant thymoma; four years later, he developed RI-SVCS and was managed with $10 \mathrm{~mm}$ ringed polytetrafluoroethylene grafting between the left internal jugular and left femoral veins.
Percutaneous transluminal angioplasty (PTA) with stenting is commonly considered to be an accepted management of SVCS from malignant causes, along with radiation therapy and/or chemotherapy (9). The management of SVCS from benign causes has traditionally used surgical bypass grafting of the SVC, which is believed to provide lower rates of reocclusion (9). However, a series of 16 patients with benign SVCS treated with thrombolysis and PTA with stenting reported an $85 \%$ patency rate at 17 months mean follow-up (10), while a larger study of 32 patients with benign SVCS treated surgically identified a 79\% graft patency after mean follow-up of 5.3 years (9). It has been suggested in a recent review that benign SVCS should be managed with PTA and stenting, and surgical management reserved for those who fail this approach (11). It should be noted that most cases of benign SVCS reported in these two case series resulted from either intravascular thrombosis related to indwelling central venous catheters or cardiac rhythm management devices.

\section{CONCLUSION}

We report a case of chemoradiation-induced SVCS in a patient achieving local control of his lung cancer based on thoracic imaging and operative/pathological findings. Dosimetric and volume parameters would not have predicted this outcome given the routine volume and V20Gy treated in the present case. The management of benign SVCS cases can be controversial, because these patients typically have longer survival than those with malignant SVCS. Based on our literature review, reported cases of chemotherapy-induced and RI-SVCS have been managed surgically with endovascular interventions or by anticoagulation.

\section{REFERENCES}

1. Yellin A, Rosen A, Reichert N, Lieberman Y. Superior vena cava syndrome. The myth - the facts. Am Rev Respir Dis 1990;141:1114-8.

2. Fajardo LF, Berthrong M, Anderson RE. Radiation Pathology, 1st edn. New York: Oxford University Press, 2001.

3. Delanian S, Lefaix JL. The radiation-induced fibroatrophic process: Therapeutic perspective via the antioxidant pathway. Radiother Oncol 2004;73:119-31.

4. Denham JW, Hauer-Jensen M. The radiotherapeutic injury a complex 'wound'. Radiother Oncol 2002;63:129-45.

5. Turk HM, Camci C, Buyukberber S, et al. Superior vena cava syndrome caused by chemotherapy-induced fibrosis. J Chemother 2002;14:417-9.

6. Van Putten JW, Schlosser NJ, Vujaskovic Z, Leest AH, Groen HJ. Superior vena cava obstruction caused by radiation induced venous fibrosis. Thorax 2000;55:245-6.

7. Lee Y, Doering R, Jihayel A. Radiation-induced superior vena cava syndrome. Tex Heart Inst J 1995;22:103-4.

8. Dhaliwal RS, Das D, Luthra S, Singh J, Mehta S, Singh H. Management of superior vena cava syndrome by internal jugular to femoral vein bypass. Ann Thorac Surg 2006;82:310-2.

9. Kalra M, Gloviczki P, Andrews JC, et al. Open surgical and endovascular treatment of superior vena cava syndrome caused by nonmalignant disease. J Vasc Surg 2003;38:215-23.

10. Kee ST, Kinoshita L, Razavi MK, Nyman URO, Semba CP, Dake MD. Superior vena cava syndrome: Treatment with catheterdirected thrombolysis and endovascular stent placement. Radiology 1998;206:187-93.

11. Schifferdecker B, Shaw JA, Piemonte TC, Eisenhauer AC. Nonmalignant superior vena cava syndrome: Pathophysiology and management. Catheter Cardiovasc Interv 2005;65:416-23. 


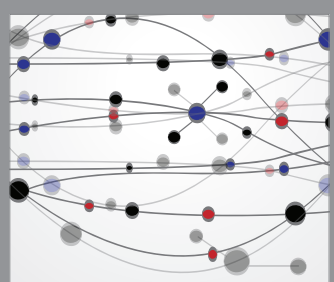

The Scientific World Journal
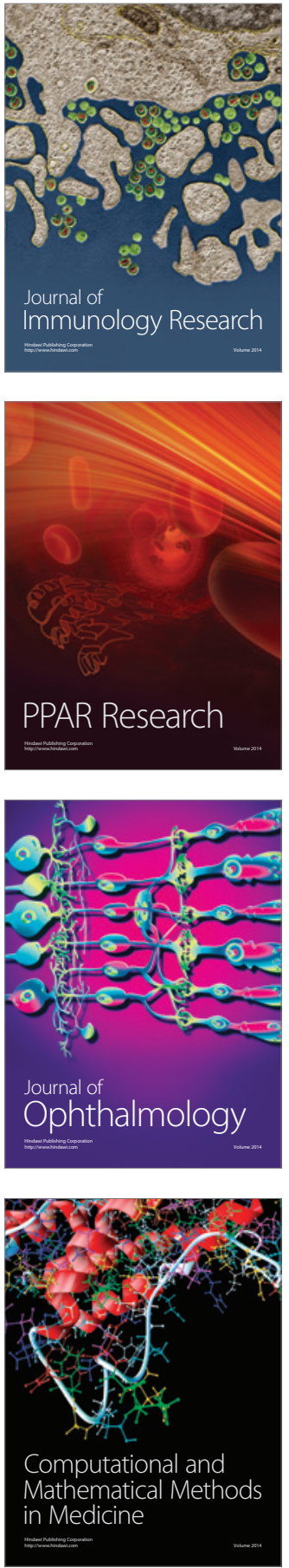

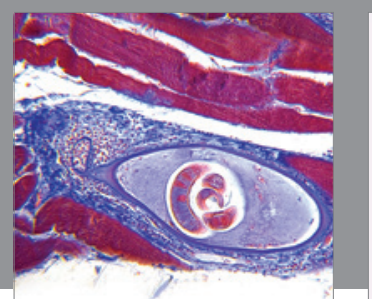

Gastroenterology Research and Practice

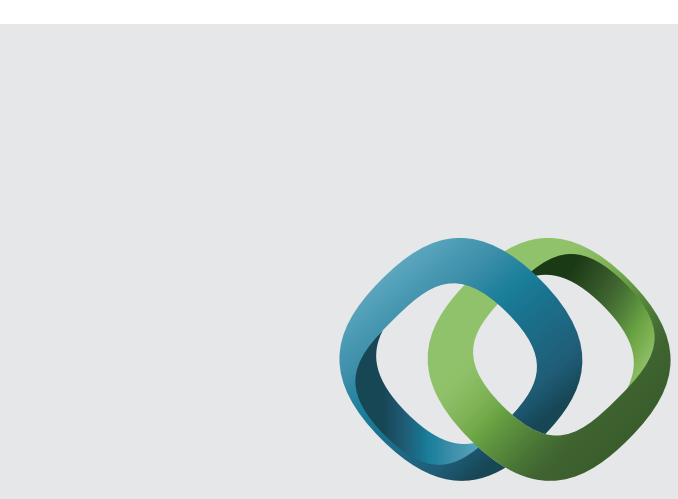

\section{Hindawi}

Submit your manuscripts at

http://www.hindawi.com
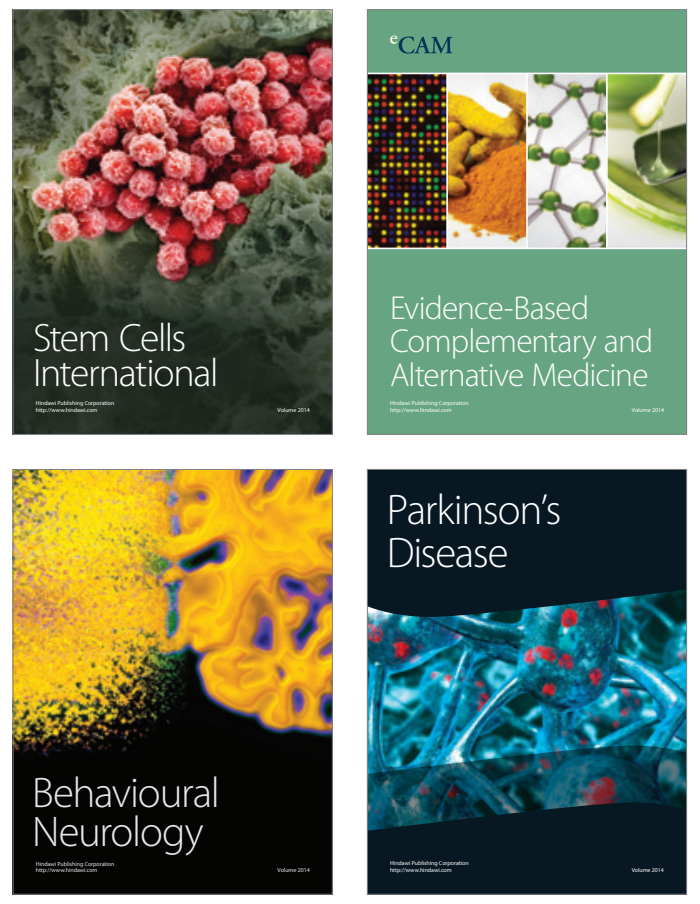
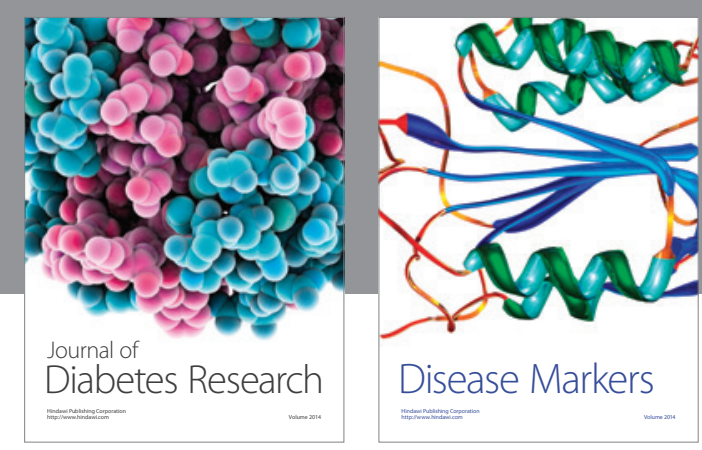

Disease Markers
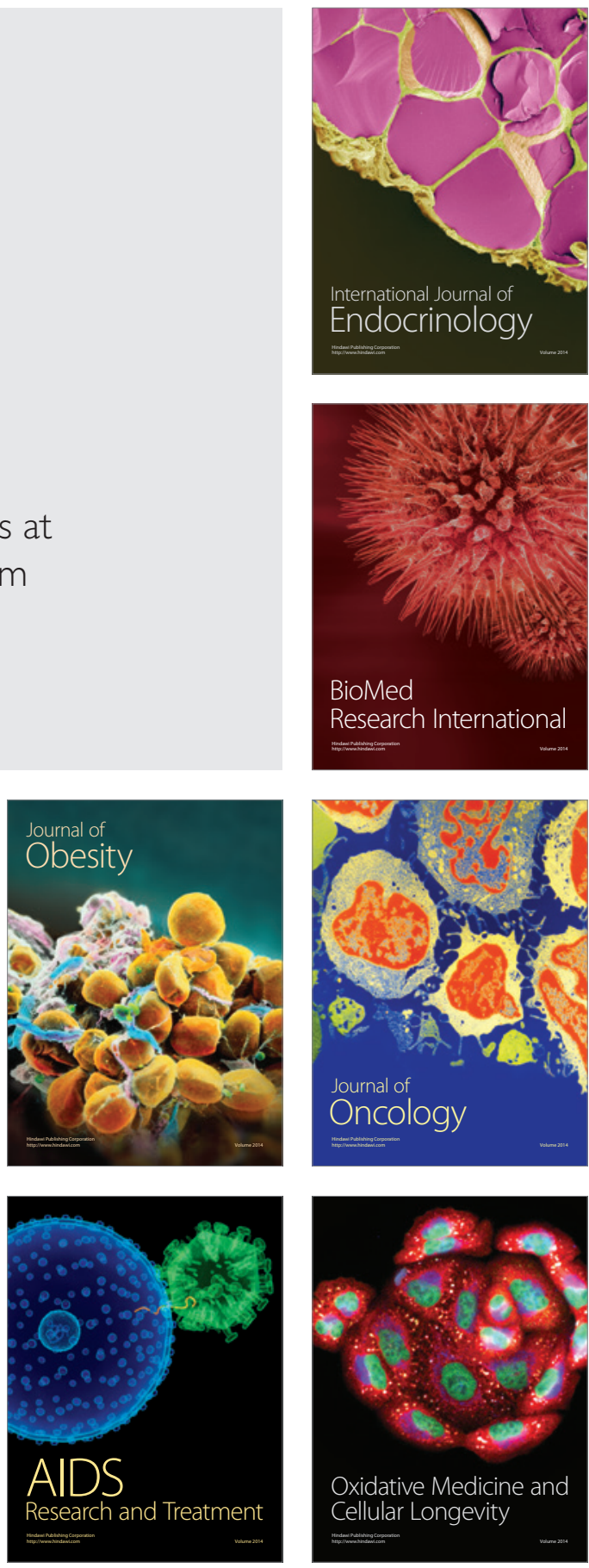